\title{
Optimization of Varying Orientation of Continuous Fiber Direction and Its Applications to New Methods of Additive Manufacturing
}

\author{
Luca De Vivo Nicoloso* \\ Department of Structural Engineering, USA \\ *Corresponding author: Luca De Vivo Nicoloso, Department of Structural Engineering, USA

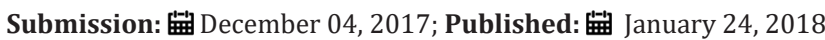

\begin{abstract}
The main purpose of this paper is to study the opportunities of structural composite material optimization using additive manufacturing, in specific the optimization of varying orientation of continuous fiber direction in composite structures. Recent advances in additive manufacturing, with the introduction of fused deposition modeling of continuous fiber-reinforced thermoplastics, have opened a door for further improvements in composite manufacturing. Optimal orientation of fibers can improve structural stiffness, strength, ultimate failure load, buckling stress, and fundamental frequency of composite laminates. A new technique for composite manufacturing, combining additive manufacturing with advanced composites using a multi-axis robotic arm, opens the door for new optimization methods where composite structures are not only optimize in the in-plane direction but also in the vertical axis. This is believed to yield parts that are stronger, safer, and lighter.
\end{abstract}

\section{Introduction}

In recent years the use of composite laminated plates have been widely adopted by the engineering community especially in practices such as aerospace, marine, civil and medical engineering. Composite materials are made by combining two or more materials, which often have different properties, in order to create a material with unique properties. One of the most widely used composite materials is fiber-reinforced composite (FRC), where fibers and a matrix are used to create structures with an exceptional strength to weight ratio. The fiber provides strength in the longitudinal direction while the matrix, often a resin, provides stiffness and bonding of the fibers. A special characteristic of FRC is that multiple plys with different fiber orientations can be stacked in order to provide strength in multiple directions, which makes composite plates a material that can be tailored for specific applications or load cases.

Extensive literature can be found in which researchers have study several ways of optimizing composite structures with respect to multiple fiber variables such as; fiber's materials [1], volume fraction [2,3], size [4] and mesostructured. In fiber-matrix composite material mechanics the orientation of the fibers plays an essential role in dictating the performance of the composite material. This implies that there is an opportunity to optimize the orientation of the fibers to obtain composite structures that outperform unidirectional fiber lay-ups. Studies have shown that in-plane optimal fiber orientation can increase structural stiffness [5-7], failure loading [8-10] and buckling stress [11-13], and improve the fundamental frequency of the laminate [14-17] over the traditional quasi-isotropic fiber construction without increasing the weight.

Early studies to describe the behavior of composite plates with variable fiber spacing, and fiber orientation were conducted [18-22]. Marting AF \& Leissa AW [18] studied the buckling of a rectangular composite sheet composed of variably spaced straight and parallel fibers (variable fiber volume fraction), and achieved improvement in the buckling performance. Kuo CM et al. [19] modeled elastomer composite sheets having fibers in the form of sinusoidal waves. Hyer MW \& Lee HH [20] developed finite element models of panels with curvilinear fiber format to improve strength and buckling performance [19]. Dinardo MT \& Lagace PA [21] found that for panels with dropped internal plies the buckling performance is driven by the changes in stiffness along the panel. Gurdal Z \& Olmedo R [22] carried out studies of rectangular panels with steered fiber path and was able to obtain solutions to the plane elasticity problem, and create the necessary equations to generate the linear variation of fiber orientation angles.

Further improvements on the understanding of the behavior and benefits of varying curvilinear fibers in composite structures were performed at the Karlsruhe Institute of Technology in Germany. Mattheck C and Kubler et al. [23,24] studied the biomechanics of wood and bone structures. They found that trees 
optimize their external shape such that stress concentrations are avoided. Their studies on natural load carriers have demonstrated that shear stresses, which are extremely dangerous for fiber composite materials, are nearly eliminated by a force flow-tailored orientation of the fibers. Thus, the risk of splitting and formation of shear cracks, which may lead to failure of fiber composites, is averted. After these findings Reuschel D et al. [10] developed the CAIO-Method which uses Finite Element Method (FEM) analyses and copies biological fiber orientation in order to minimize shear stresses within orthotropic or multi-layer composite material structures. The idea behind CAIO Method is to lay the fibers in the direction of the principal stresses. The optimum fiber arrangement is calculated by an iterative process of alternating FE-run and CAIO-calculation. The method is characterized by an excellent convergence. Most of the times three or four iterations are enough to reduce the shear stresses by more than $90 \%$.

As mentioned earlier spacial variation of stiffness can be induced by steering composite fibers in curvilinear paths. Fiber angles and predefined fiber angle variations are used in most of the research on fiber steered composites [25]. However, it has been proposed to design such variable stiffness structures in terms of lamination parameters. The use of lamination parameters efficiently defines stiffness variation over a structural domain with the minimum number of variables. Van Campen et al. [25] proposed a two step design approach, where first a Variable Stiffness composite is designed in terms of Lamination Parameters, followed by a conversion of the lamination parameters into fiber angle distribution for each layer in the laminate.

A common objective function in structural optimization is the minimization of compliance, which is also referred to as the strain energy. The compliance of a structure is essentially the inverse of stiffness, and by minimizing compliance, one can maximize the stiffness. Hammer, Bendsoe, Lipton and Pedersen et al. considered the maximal stiffness design of constant stiffness laminated plates subjected to single and multiple loads, where the stiffness of the laminates were parametrized in terms of the lamination parameters. Setoodeh et al. [5] used lamination parameters to investigate the optimal design of fiber reinforced rectangular composite plates for minimum compliance. They considered two types of designs: constant-stiffness and variable-stiffness, and used a sequential quadratic programming to solve the optimization problem. It was shown that significant improvements in stiffness can be gained by using variable-stiffness design. Brampton J et al. [6] proposed a new optimization method to minimize the compliance of a structure by optimizing the continuously varying fiber paths using a level set method. They derived the sensitivity of the structural compliance to a change of fiber direction, and use the sensitivities to optimize the level set defined fiber paths. Setoodeh et al. [7] investigated the design optimization of variable-stiffness composite layers using cellular automata. The analysis is based on the equilibrium of local cells and the design process uses the compliance minimization as the objective function. They were able to show that substantial gains in compliance can be achieved through placement of fibers in their optimal spatial configuration.
Another important optimization problem is the maximization of strength, or the maximization of failure load. The strength characteristics of a composite lamina are generally just as important as the material stiffness of compliance characteristics. The challenge behind it is that strength properties vary as a function of load direction (compression or tension) and load type (longitudinal, transverse normal, or shear) relatively to the material orientation. The strength of a lamina material can be determined through micro-mechanics equations and are defined in the material principal direction. Failure theories have been developed for lamina assuming biaxial stress state, where the strength properties are defined in the material principal directions, and thus the stresses must be transformed not to the principal stress axes but to the material axes. The maximum failure criterion can be adopted to predict failure load and the failure type (fiber failure, matrix failure, or shear failure) of a single ply. In addition to the simple strength of material approaches, other methods have been developed based upon the energy distorted within the specimen at failure. These methods allow the use of a single simple equation that can be used during preliminary design and analysis to predict the failure load of the lamina. Some of the most common energy distortion methods are Tsai-Hill, Hoffman, and Tsai-Wu failure criterions. We found that Tsai-Wu is a preferred failure criterion used by the composite optimization community. Huang et al. [8] proposed a procedure for optimizing the fiber orientation near a hole in a single layer of multilayer composite laminates for increased strength. The Tsai-Wu failure criterion was used and an optimization of the fiber direction near the hole was obtained by alternating between a gradient based search and a genetic algorithm. As mentioned earlier Reushcel et al. [10] created the CAIO method which copies biological fibre orientation in order to minimize shear stresses within composite layers, thus increasing the strength of the lamina. Temmen $\mathrm{H}$ et al. [9] presented selected results of four research projects, carried by the DLR Institute of Composite Structures and Adaptive Systems, which aimed to improve the design of composites using Tailored Fibre Placement by aligning the fibre to the direction of principal stresses for a given load case. They were able to achieve improvements of about $60 \%$ for tension and $79 \%$ for compression.

Because composite material specimens tend to be relatively thin, buckling prior to the desired failure mode is a concern when specimens are loaded in compression or bending. Known as Euler buckling, the test specimen carries the applied load as it increases with no indication of incipient failure until it suddenly collapses when the load reaches a critical value. Karakaya et al. [12] studied the optimization of stacking sequence of laminated composite plates for maximum buckling capacity using genetic algorithm and generalized pattern search algorithm. Ho-Huu et al. [26] proposed a novel numerical optimization method to maximize the buckling load of a constant-stiffness laminated composite plates using improved differential evolution and smoothed finite element method. The design variables used were fibre orientation angles and thickness of layers. Van Den Brink et al. proposed a two-step variable stiffness design approach for buckling optimization of a composite fuselage window section. First the optimal fibre orientation is determined 
based on spline interpolation and then the physical composite fibre tows are derived. An improvement of $12 \%$ in buckling performance was found when compared to constant stiffness laminate. Abdalla et al. [11] worked on the optimization of variable stiffness panels for maximum buckling load using lamination parameters. Numerical results demonstrated improvements in excess of $100 \%$ in buckling loads of variable stiffness panels compared to the optimum constant stiffness designs.

The fundamental frequency of composite laminated plates has been considered as an important factor in vibration problems. Aeronautic structures generally require that the fundamental frequency to be higher than those of the excitations. If this requirement is practically unattainable, then the fundamental frequency is required to lie between two excitation frequencies. Koide R \& Luersen M [13] proposed the use of an ant colony algorithm to maximize the fundamental frequency of laminated composite cylindrical shells. It Sadr et al. [14] presented a new method to optimize constant-stiffness composite plates for maximum fundamental frequency using a combination of Elitist Genetic algorithm and finite strip method. Wang F et al. [15] worked on the fiber orientation angles optimization for maximum fundamental frequency of a constant stiffness laminated composite plates using a Generic Algorithm and a Meshless Method. Fukunaga et al. [16] studied optimal design of constant stiffness laminates for natural frequency using 4 lamination parameters based on a CLPT (Classical Lamination Plate Theory) deformation analysis. Abdalla et al. [17] considered the maximization of the natural frequency of variable stiffness composite panels using lamination parameters by adopting a new structural approximation scheme, the so called generalised reciprocal approximation, which leads to a robust stable convergence of the design iteration. They were able to prove that significant increase in the optimal fundamental frequency can be achieved using variable stiffness panels.

Recent improvements in the area of additive manufacturing allows the production of complex arrangement of fibers using a technique known as Fused Deposition Modeling (FDM) of continuous carbon fibre reinforced thermoplastic (CCFT). Automated fiber placement of thermoset composites was invented over 40 years ago and is now widely accepted for the manufacture of advanced composite structures. However, expensive tooling and post process such as autoclaves are required. In-situ inexpensive tooling, eliminates these post process, and can be considered an additive manufacturing process for advanced composite structures. Matsuzaki R \& Ueda M [27] developed a method for three-dimensional (3D) printing of continuous fiber-reinforced thermoplastics by in-nozzle impregnation [28]. A thermoplastic filament and continuous fibers are separately supplied to the 3D printer and the fibers were impregnated with the filament within the heated nozzle of the printer immediately before printing. Another technique proposed by MarkForged is to alternate inplane layers of thermoplastic and fiber materials. A limitation of these methods is that the strength of the $\mathrm{Z}$ axis is restricted to the interlayer bonding strength. Turk DA et al. [8] proposed to use FDM together with a multi-axis robot to place continuous fiber in three dimensions, and build structures from the inside out. This can be achieved by combining additive manufacturing tooling with insitu automated fiber placement of thermoplastic composite rapid prototyping software with Automated Fiber Placement. Yamanaka et al. [29] worked on the fiber line optimization in single ply for 3D printed composites. They optimization of the in-plane curved carbon fiber placement in a planar ply with an open hole. The project dealt with the in-plane strength maximization of a curved carbon fibre alignment of a ply with an open hole using genetic algorithm.

\section{Conclusion}

In this paper we investigated previous attempts to optimize composite structures by using a technique known as variable stiffness. We found that in-plane optimal fiber orientation can increase structural compliance, failure loading, and buckling stress, and improve the fundamental frequency of the structure. In multiple studies it was shown that significant increase in desired structural performance can be achieved using variable stiffness panels when compared with optimal constant stiffness ones. We also found that a new technique for composite manufacturing, combining additive manufacturing with advanced composites using a multi-axis robotic arm, opened the door for new optimization methods where composite structures are not only optimize in the in-plane direction but also in the vertical axis. This is believed to yield parts that are stronger, safer, and lighter when compared with structures that are optimized in the in-plane axes only. Limited literature was found when dealing with this type of problems and we believe this field offers great opportunity for future research.

Moving forward we want to study the optimization of inplane continuous fiber direction on multiple plys laminate. We believe that by doing this we can optimize the in-plane behavior of a composite plate exposed to multiple loads. We would also like to further study the capabilities of new composite additive manufacturing and optimization methods to allow the exploitation of its full capacities in structural engineering. Of principal interest is the study of a natural structure, the cholla cactus (Figure 1), and the combination of topology and fiber orientation optimization to understand this structure and to design structures alike.

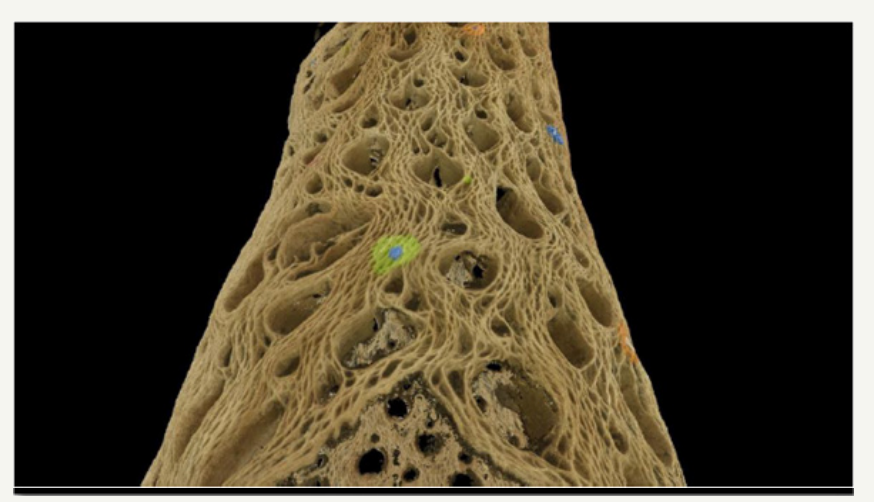

Figure 1: 3D reconstruction of a Cholla cactus skeleton using structure from motion. 


\section{References}

1. Chen L, Zhang Y (2013) A study on the application of material selection optimization approach for structural-acoustic optimization. Materials \& Desig 52: 207-213.

2. Kyung SN, Kim JH (2009) Volume fraction optimization of functionally graded composite panels for stress reduction and critical temperature. Finite Elem Anal Des 45(11): 845-851.

3. Domnica SM, Ioan C, Ionut T (2016) Structural optimization of composite from wind turbine blades with horizontal axis using finite element analysis. Procedia Technology 22: 726-733.

4. El-Shekeil YA, Sapuan SM, Azaman MD, Jawaid M (2013) Optimization of blending parameters and fiber size of kenaf-bast-fiber-reinforced the thermoplastic polyurethane composites by taguchi method. Advances in Materials Science and Engineering.

5. Setoodeh S, Abdalla MM, Gürdal Z (2006) Design of variable stiffness laminates using lamination parameters. Composites, Part B: Engineering 37(4-5): 301-309.

6. Christopher JB, Chauncey Wu K, Alicia Kim H (2015) New optimization method for steered fiber composites using the level set method. Struct Multidiscip Optim 52(3): 493-505.

7. Setoodeh S, Gürdal Z, Watson LT (2006) Design of variable-stiffness composite layers using cellular automata. Computer Methods in Applied Mechanics and Engineering 195(9-12): 836-851.

8. Huang J, Haftka RT (2005) Optimization of fiber orientations near a hole for increased load carrying capacity of composite laminates. Structural and Multidisciplinary Optimization 30(5): 335-341.

9. Temmen H, Degenhardt R, Raible T (2006) Tailored fibre placement optimization tool. Proceedings of the $25^{\text {th }}$ Congress of the International Council of the Aeronautical Sciences 4: 2462-247.

10. Reuschel D, Mattheck C, Teschner M (1997) Determination of optimal fibre arrangement of complex two or three dimensional geometries. Computer aided optimum design of structures, Proceedings of OPTI, Rome.

11. Ijsselmuiden ST, Abdalla MM, Gürdal Z (2010) Optimization of variable-stiffness panels for maximum buckling load using lamination parameters. AIAA Journal 48(1): 134-143.

12. Karakaya Ş, Soykasap Ö (2009) Buckling optimization of laminated composite plates using genetic algorithm and generalized pattern search algorithm. Structal Multidisciplinary Optimization 39: 477.

13. Koide R, Luersen M (2013) Maximization of fundamental frequency of laminated composite cylindrical shells by ant colony algorithm. Journal of Aerospace Technology and Management 5(1).

14. Sadr MH, Bargh GH (2012) Optimization of laminated composite plates for maximum fundamental frequency using elitist-genetic algorithm and finite strip method. Journal of Global Optimization 54(4): 707-728.
15. Wang F, Zhao WP, Xiang S (2015) Fiber orientation angles optimization for maximum fundamental frequency of laminated composite plates by the genetic algorithm and meshless method. Applied Mechanics and Materials 709: 130-134.

16. Fukunaga H, Sekine H, Sato M (1994) Optimal design of symmetric laminated plates for fundamental frequency. Journal of Sound and Vibration 171(2): 219-229.

17. Abdalla MM, Setoodeh S, Gurdal Z (2007) Design of variable stiffness composite panels for maximum fundamental frequency using lamination parameters. Composite Structures 81(2): 283-291.

18. Marting AF, Leissa AW (1989) Application of the ritz method to plane elasticity problems for composite sheets with variable fiber spacing. International Journal of Numerical Methods in Engineering 28(8): 18131825 .

19. Kuo CM, Takahashi H, Chou TW (1988) Effect of fiber waviness on the nonlinear elastic behavior of flexible composites. Journal of Composite Materials 22(11): 1004-1022.

20. Hyer MW, Lee HH (1991) The use of curvilinear fiber format to improve buckling resistance of composite plates with central circular holes. Composite Structures 18(3): 239-261.

21. Dinardo MT, Lagace PA (1989) Buckling and postbuckling of laminated composite plates with ply drop-offs. AIAA Journal 27(10): 1392-1398.

22. Gurdal Z, Olmedo R (1993) In-Plane response of laminates with spatially varying fiber orientations: variable stiffness concept. AIAA Journal 31(4).

23. Mattheck C (1988) Design in nature-Learning from tree, Springer Verlag, Germany.

24. Mattheck C, Kubler H (1997) Wood-The internal optimization of trees, Springer Verlag, Berlin, ( $\left.2^{\text {nd }} e d n\right)$, Germany.

25. Julien MJ, Van Campen, Kassapoglou C, Gürdal Z (2012) Generating realistic laminate fiber angle distributions for optimal variable stiffness laminates. Composites Part B: Engineering 43(2): 354-360.

26. Ho-Huu V, Do-Thi TD, Dang-Trung H, Vo-Duy T, Nguyen-Thoi T (2016) Optimization of laminated composite plates for maximizing buckling load using improved differential evolution and smoothed finite element method. Composite Structures 146: 132-147.

27. Matsuzaki R, Ueda M (2016) Three-dimensional printing of continousfiber composites in-nozzle impregnation. Scientific Reports 6.

28. Türk DA, Triebe L, Meboldt M (2016) Combining additive manufacturing with advanced composites for highly integrated robotic structures. CIRP 50: 402-407.

29. Yamanaka Y, Todoroki A, Ueda M, Hirano Y, Matsuzaki R (2016) Fiber line optimization in single ply for 3D printed composites. Open Journal of Composite Materials 6(4): 121-131. 